\title{
Exponential Stability \\ of a \\ Nonlinear Distributed Parameter System
}

\author{
J. Tervo and M. Nihtilä
}

\begin{abstract}
A nonlinear parabolic partial differential equation model describing the behaviour of a distributed parameter fixed-bed bioreactor is studied here. Exponential stability around the steady state solution for exponentially decaying deviations in the input and disturbance are proved via abstract formulation of the model as an evolution equation and by utilizing semigroup theory and asymptotic stability of the corresponding evolution operator.
\end{abstract}

Keywords: Infinite-dimensional nonlinear systems, parabolic partial differential equations, exponential stability, fixed-bed bioreactors

AMS subject classification: Primary $93 \mathrm{C} 20$, secondary $93 \mathrm{C} 80,35 \mathrm{~K} 57$

\section{Introduction}

Several different stability concepts have been developed for linear as well for nonlinear partial differential equations and the corresponding evolution operators (see, e.g., Amann [2: p. 68], Lions [14: p. 172], and Curtain and Zwart [5: p. 215]). As compared with the stability of finite-dimensional systems we are facing more complicated situations. Even in the case of linear partial differential equations the location of the poles of the transfer function of the distributed parameter system does not determine directly the stability as is the case of finite-dimensional systems. More refined funtional-analytic and function-space tools are needed. Moreover, the variety of different partial different equation system models is larger depending, e.g. on the location of inputs and outputs, and on the corresponding operator classes defined on abstract function spaces (Banach or Hilbert spaces).

Here, we consider stability properties of solutions of a nonlinear system related to a distributed parameter fixed-bed bioreactor. The system is a infinite-dimensional one. It is governed by partial differential equations of parabolic type, i.e. they are so-called evolution equations (c.f., e.g., Tanabe [21]).

The distributed parameter model of the system has its background in biological water treatment processes $[7,8,13]$. The goal of the process is to remove harmful nitrogen compounds from drinking water or from communal waste water. The process

J. Tervo: Dept. Appl. Math. Univ., POB 1627, FIN-70211 Kuopio M. Nihtilä: Dept. Food Techn. Univ., POB 27 (Viikki), FIN-00014 University of Helsinki

ISSN 0232-2064 / \$2.50 (c) Heldermann Verlag Berlin 
is modelled by two coupled partial differential equations. They describe the growth and substrate (nitrogen) consumption of certain microorganisms. These are immobilised on a fixed bed in the reactor tube. The water to be treated and which includes the substrate flows through the reactor.

The spatially one-dimensional model of the fixed-bed bioreactor consists of a pair of nonlinear partial differential equations

$$
\left.\begin{array}{l}
\frac{\partial u_{1}}{\partial t}=-k_{d} u_{1}+\mu\left(u_{1}, u_{2}\right) u_{1} \\
\frac{\partial u_{2}}{\partial t}=D \frac{\partial^{2} u_{2}}{\partial x^{2}}-c(t) \frac{\partial u_{2}}{\partial x}-k_{1} \mu\left(u_{1}, u_{2}\right) u_{1}
\end{array}\right\}
$$

where the spatial variable $x$ belongs to the interval $G=(0,1) \subset \mathbb{R}$ and the evolving time $t$. belongs to the interval $\mathbb{R}_{+}=(0, \infty)$ (remark that by $\overline{\mathbb{R}}_{+}$the interval $[0, \infty)$ is denoted). The boundary conditions applied here are due to Danckwerts [6] and they are of the form

$$
\left.\begin{array}{l}
\frac{\partial u_{2}}{\partial x}(0, t)=\frac{c(t)}{D}\left(u_{2}(0, t)-S(t)\right) \\
\frac{\partial u_{2}}{\partial x}(1, t)=0
\end{array}\right\} \quad\left(t \in \mathbb{R}_{+}\right)
$$

In the equations the states $u_{1}=u_{1}(x, t)$ and $u_{2}=u_{2}(x, t)$ are the concentrations of the biomass of the microorganisms and the substrate, respectively. The specific growth rate of the microorganisms (in biomass)

$$
\mu\left(u_{1}, u_{2}\right)=\mu_{m} \frac{\dot{u}_{2}}{k_{2} u_{1}+u_{2}}
$$

is due to Contois, 1959. This makes system (1) nonlinear. The input flow $c$ is the control variable and the input substrate concentration $S$ is a disturbance variable in the system. They are generally smooth functions of time, i.e. $c=c(t)$ and $S=S(t)$. The output function $y$ (the measured variable) is the substrate concentration at the end of the reactor, that is,

$$
y(t)=u_{2}(1, t)
$$

The initial condition

$$
\left.\begin{array}{l}
u_{1}(x, 0)=u_{10}(x) \\
u_{2}(x, 0)=u_{20}(x)
\end{array}\right\}
$$

is chosen in such a way that $u_{10}$ and $\dot{u}_{20}$ are the steady state solutions of problem (1) - (2) before the simulated step changes the input function $c(t)$ and/or the the initial concentration $S(t)$ of the substrate. In the steady state $c$ and $S$ are independent of time and in that case they are denoted by $\bar{c}$ and $\bar{S}$. Other parameters are positive. We do not list their meanings here but we refer to the contributions $[7,8]$, where the model and the parameters from the system-theoretic point of view are explained in more detail.

Simulation studies carried out pointed out that the assumption of equally distributed concentrations on the constant cross-sectional area of the reactor tube was adequate (c.f. 
$[17,23])$, justifying the use of the single space variable, the scaled distance from the input of the reactor tube (the length of which is scaled equal to 1). Boundary conditions, which are different from (2), have been proposed for dispersion models of our type (c.f. [20]), and applied in [13].

In Section 2 problem (1) - (3) is put into the abstract form

$$
\left.\begin{array}{rl}
\dot{v}+A(t) v & =F(t, v) \\
v(0) & =v_{0}
\end{array}\right\}
$$

where $A(t)$ is a linear unbounded operator in appropriate Banach spaces and $F(t, v)$ is a nonlinear function. This enables us to consider the existence, stability and other issues of solutions as an application of the semigroup theory (see, e.g., $[1,2,5,9,10$, $18,21])$. The existence of a positive classical global solution $u=\left(u_{1}, u_{2}\right)$ of problem (1) - (3) such that

$$
\left.\begin{array}{l}
u_{1} \in C^{1}((0, \infty), C(\bar{G})) \cap C\left(\overline{\mathbb{R}}_{+}, C(\bar{G})\right) \\
u_{2} \in C^{1}((0, \infty), C(\bar{G})) \cap C\left((0, \infty), C^{2}(\bar{G})\right) \cap C\left(\overline{\mathbb{R}}_{+}, C(\bar{G})\right)
\end{array}\right\}
$$

was proved in [11] as an application of the semigroup theory. Numerical computations for the original nonlinear system in the state space seem to support analytical stability result obtained here, not only in the spatially 1-dimensional but also in the spatially 3-dimensional case $[17,23]$.

In Section 3 we show that the steady state solution of problem (1) - (3) is attractive. The proof is based on the fact that the evolution operator $U(t, \tau)$ of the operator $A(t)$ can be shown to be asymptotically stable in the relevant spaces considered here. The asymptotic stability of $U(t, \tau)$ follows from the results for quasilinear parabolic equations on the interpolation-extrapolation spaces studied in detail in [2]. It is well-known that exponential stability of the solutions both in finite-dimensional and infinite-dimensional cases influences on the input-output stability of the system (c.f. [5]). Consequently, it is a central issue of our system study for control.

We give some preliminary notations applied here:

Let $G$ be an open set in $\mathbb{R}^{n}$. $C(\bar{G})$ is the space of continuous functions on $\bar{G}$ equipped with the norm $\|w\|_{C(\bar{G})}\left(=\|w\|_{\infty}\right)=\sup _{x \in \bar{G}}|w(x)|$. Further, $L_{p}(G) \quad(1 \leq p<\infty)$ is the Lebesgue space of $p^{\text {th }}$-power integrable functions $f: G \mapsto C$ and $W^{s, p}(G)$ (s $\epsilon$ $\mathbb{R}, 1 \leq p<\infty)$ is the Sobolev-Slobodeckii space (see [2]).

Let $\Delta$ be an interval in $\mathbb{R}$. Then $C^{\prime}(\Delta, X)$ is the space of all $l$ times continuously differentiable functions $f: \Delta \mapsto X$, when $X$ is a normed space. The space $C^{\rho-}(\Delta, X) \quad(0<\rho \leq 1)$ is the space of Hölder continuous functions $f: \Delta \mapsto X$ equipped with the usual norm (see, e:g., [2: p. 40]). In the product space $X_{1} \times X_{2}$ of Banach spaces $X_{1}$ and $X_{2}$ we use the norm $\left\|\left(w_{1}, w_{2}\right)\right\|_{X_{1} \times X_{2}}=\left\|w_{1}\right\|_{X_{1}}+\left\|w_{2}\right\|_{X_{2}}$. 


\section{Abstract formulation of the partial differential equation system}

The original partial differential equation problem (1) - (3) will be converted into the abstract form

$$
\left.\begin{array}{rl}
\dot{v}+A(t) v & =F(t, v) \\
v(0) & =v_{0}
\end{array}\right\}
$$

where $A(t)$ is a linear unbounded operator and $F(t, v)$ is quadratically bounded by $v$. This means that the linearized version of the original partial differential equation problem is given by

$$
\left.\begin{array}{rl}
\dot{v}+A(t) v & =F(t, 0) \\
v(0) & =v_{0}
\end{array}\right\}
$$

2.1 Preliminaries. Consider the problem

$$
\left.\begin{array}{l}
\frac{\partial u_{1}}{\partial t}=f\left(u_{1}, u_{2}\right)-k_{d} u_{1} \\
\frac{\partial u_{2}}{\partial t}=D \frac{\partial^{2} u_{2}}{\partial x^{2}}-c(t) \frac{\partial u_{2}}{\partial x}-k_{1} f\left(u_{1}, u_{2}\right)
\end{array}\right\}
$$

for $(x, t) \in G \times \mathbb{R}_{+}$,

$$
\left.\begin{array}{l}
\frac{\partial u_{2}}{\partial x}(0, t)=\frac{c(t)}{D}\left(u_{2}(0, t)-S(t)\right) \\
\frac{\partial u_{2}}{\partial x}(1, t)=0
\end{array}\right\}
$$

for $t \in \mathbb{R}_{+}$, and

$$
u(x, 0)=u_{0}(x)=\left(u_{10}(x), u_{20}(x)\right)
$$

for $x \in G$. Above we denoted

$$
G=(0,1) \quad \text { and } \quad f\left(u_{1}, u_{2}\right)=\mu\left(u_{1}, u_{2}\right) u_{1}=\mu_{m} \frac{u_{1} u_{2}}{k_{2} u_{1}+u_{2}}
$$

Occasionally we also use the notation

$$
f(u)=f\left(u_{1}, u_{2}\right) \quad \text { for } u=\left(u_{1}, u_{2}\right) .
$$

Throughout the paper we assume that $c$ and $S$ are positive $C^{1}\left(\overline{\mathbb{R}}_{+}\right)$-functions and that $\dot{c}$ and $\dot{S}$ are locally Lipschitz continuous. In addition we assume that $u_{10}$ and $u_{20}$ are positive $C^{1}(\bar{G})$-functions.

Let $\bar{u}_{1}$ and $\bar{u}_{2}$ be solutions of the steady state equations

$$
\left.\begin{array}{l}
0=f\left(\bar{u}_{1}, \bar{u}_{2}\right)-k_{d} \bar{u}_{1} \\
0=D \frac{\partial^{2} \bar{u}_{2}}{\partial x^{2}}-\bar{c} \frac{\partial \bar{u}_{2}}{\partial x}-k_{1} f\left(\bar{u}_{1}, \bar{u}_{2}\right)
\end{array}\right\}
$$


for $S(t)=\bar{S}$ and $c(t)=\bar{c}$, for $x \in G$, with the two-point boundary conditions

$$
\left.\begin{array}{l}
\frac{\partial \bar{u}_{2}}{\partial x}(0)=\frac{\bar{c}}{D}\left(\bar{u}_{2}(0)-\bar{S}\right) \\
\frac{\partial \bar{u}_{2}}{\partial x}(1)=0
\end{array}\right\}
$$

where $\bar{c}=c(0)$. The solutions $\bar{u}_{1}$ and $\bar{u}_{2}$ can be computed in closed form and they have the expressions

$$
\left.\begin{array}{l}
\bar{u}_{2}(x)=S \frac{q \cosh (q(1-x))+p \sinh (q(1-x))}{q \cosh (q)+(a+p) \sinh (q)} e^{p x} \\
\bar{u}_{1}(x)=\frac{\mu_{m}-k_{d}}{k_{2} k_{d}} \bar{u}_{2}(x)
\end{array}\right\}
$$

where

$$
P=\frac{\bar{c}}{D}, \quad p=\frac{P}{2}, \quad a=\frac{k_{1}\left(\mu_{m}-k_{d}\right)}{k_{2} \bar{c}}, \quad q=\sqrt{\frac{P^{2}}{4}+P a} .
$$

The pair $\left(\bar{u}_{1}, \bar{u}_{2}\right)$ is the equilibrium point of the dynamical system (5) - (7) when $c \equiv \bar{c}$ and $S \equiv \bar{S}$. The constants $P, p, a, q$ are positive for the original relevant parameter values.

Remark 1. The maximum principle for parabolic systems [19] implies the following comparison result for the solutions: Suppose that $\bar{c}$ and $\bar{S}$ are positive constants such that

$$
\left.\begin{array}{l}
c(t) \leq \bar{c} \\
S(t) \leq \bar{S}
\end{array}\right\} \quad\left(t \in \overline{\mathbb{R}}_{+}\right)
$$

Let $\bar{u}$ be the steady state solution corresponding to constant values $\bar{c}$ and $\bar{S}$ of the control $c(t)$ and disturbance $S(t)$. If $u_{0} \leq \bar{u}$, then

$$
u \leq \vec{u}
$$

This result implies especially that the solutions of problem (5) - (7) are bounded when the control $c(t)$ and the disturbance $S(t)$ are bounded. In fact, estimate (11) is natural from the physical viewpoint.

2.2 Linearization. The nonlinear problem (5) - (7) is linearized around the steady state (10). Consequently, a careful analysis for the nonlinear term $f$ has to be carried out. Formally $f$ can be linearized by using the decomposition

$$
f(\bar{u}+w)-f(\bar{u})=\frac{\partial f}{\partial u_{1}}(\bar{u}) w_{1}+\frac{\partial f}{\partial u_{2}}(\bar{u}) w_{2}+g(w)
$$

where the residual $g(w)$ must have certain appropriate properties. From (8) we get

$$
\mu\left(\bar{u}_{1}, \bar{u}_{2}\right)=\mu_{m} \frac{\bar{u}_{2}}{k_{2} \bar{u}_{1}+\bar{u}_{2}}=k_{d}
$$


which also implies the last equation in (10), i.e. $\bar{u}_{1}=\bar{u}_{2} \frac{\mu_{m}-k_{d}}{k_{2} k_{d}}$. Applying (10) and (12) we find by a routine computation that

$$
\begin{aligned}
\frac{\partial f}{\partial u_{1}}(\bar{u}) & =\mu\left(\bar{u}_{1}, \bar{u}_{2}\right)-\mu_{m} k_{2} \frac{\bar{u}_{1} \bar{u}_{2}}{\left(k_{2} \bar{u}_{1}+\bar{u}_{2}\right)^{2}} \\
& =k_{d}-\mu_{m} k_{2} \frac{\bar{u}_{2}^{2}}{\left(k_{2} \bar{u}_{1}+\bar{u}_{2}\right)^{2}} \frac{\mu_{m}-k_{d}}{k_{2} k_{d}} \\
& =\frac{k_{d}^{2}}{\mu_{m}} \\
& =: a_{1} .
\end{aligned}
$$

It can be seen also that

$$
\mu_{m} \frac{\bar{u}_{2}^{2}}{\left(k_{2} \bar{u}_{1}+\bar{u}_{2}\right)^{2}} \equiv a_{1} .
$$

Similar computations show that

$$
\frac{\partial f}{\partial u_{2}}(\bar{u})=\mu_{m} k_{2} \frac{\bar{u}_{1}^{2}}{\left(k_{2} \bar{u}_{1}+\bar{u}_{2}\right)^{2}} \equiv \frac{\left(\mu_{m}-k_{d}\right)^{2}}{\mu_{m} k_{2}}=: a_{2} .
$$

Based on these calculations the following lemma, which guarantees a successful linearization, is proved.

Lemma 1. There exist constants $C>0$ and $\delta>0$ such that

$$
f(\bar{u}+w)-f(\bar{u})=a_{1} w_{1}+a_{2} w_{2}+g(w)
$$

where $a_{1}$ and $a_{2}$ are given above and where $g(w)$ satisfies

$$
\|g(w)\|_{C(\bar{G})} \leq C\|w\|_{C(\bar{G}) \times C(\bar{G})}^{2}
$$

for all $w \in C(\bar{G}) \times C(\bar{G})$ such that $\|w\|_{C(\bar{G}) \times C(\bar{G})} \leq \delta$.

The norm abbreviations $\|\cdot\|_{1}=\|\cdot\|_{C(\bar{G})}$ and $\|\cdot\|_{2}=\|\cdot\|_{C(\bar{G}) \times C(\bar{G})}$ are used in the proof, which is given in Parts $A$ and $B$.

Proof of Lemma 1. Part A: Denote $u=\bar{u}+w$. Then by using (13) - (15) we have

$$
\begin{aligned}
\left\|f(\bar{u}+w)-f(\bar{u})-\left(a_{1} w_{1}+a_{2} w_{2}\right)\right\|_{1} \\
=\mu_{m}\left\|\frac{u_{1} u_{2}}{k_{2} u_{1}+u_{2}}-\frac{\bar{u}_{1} \bar{u}_{2}}{k_{2} \bar{u}_{1}+\bar{u}_{2}}-\left(\frac{\bar{u}_{2}^{2}}{\left(k_{2} \bar{u}_{1}+\bar{u}_{2}\right)^{2}} w_{1}+k_{2} \frac{\bar{u}_{1}^{2}}{\left(k_{2} \bar{u}_{1}+\bar{u}_{2}\right)^{2}} w_{2}\right)\right\|_{1} \\
=\mu_{m} \| k_{2} \frac{u_{1} \bar{u}_{1}}{\left(k_{2} u_{1}+u_{2}\right)\left(k_{2} \bar{u}_{1}+\bar{u}_{2}\right)} w_{2}+\frac{\bar{u}_{2} u_{2}}{\left(k_{2} u_{1}+u_{2}\right)\left(k_{2} \bar{u}_{1}+\bar{u}_{2}\right)} w_{1} \\
\quad-\left(\frac{\bar{u}_{2}^{2}}{\left(k_{2} \bar{u}_{1}+\bar{u}_{2}\right)^{2}} w_{1}+k_{2} \frac{\bar{u}_{1}^{2}}{\left(k_{2} \bar{u}_{1}+\bar{u}_{2}\right)^{2}} w_{2}\right) \|_{1} \\
\leq \mu_{m}\left\{\left\|\frac{\bar{u}_{2} u_{2}}{\left(k_{2} u_{1}+u_{2}\right)\left(k_{2} \bar{u}_{1}+\bar{u}_{2}\right)}-\frac{\bar{u}_{2}^{2}}{\left(k_{2} \bar{u}_{1}+\bar{u}_{2}\right)^{2}}\right\|_{1}\right. \\
\left.\quad+k_{2}\left\|\frac{\bar{u}_{1} u_{1}}{\left(k_{2} u_{1}+u_{2}\right)\left(k_{2} \bar{u}_{1}+\bar{u}_{2}\right)}-\frac{\bar{u}_{1}^{2}}{\left(k_{2} \bar{u}_{1}+\bar{u}_{2}\right)^{2}}\right\|_{1}\right\}\left(\left\|w_{1}\right\|_{1}+\left\|w_{2}\right\|_{1}\right) .
\end{aligned}
$$


Part B: Furthermore, we have

$$
\begin{aligned}
& \left\|\frac{\bar{u}_{1} u_{1}}{\left(k_{2} u_{1}+u_{2}\right)\left(k_{2} \bar{u}_{1}+\bar{u}_{2}\right)}-\frac{\bar{u}_{1}^{2}}{\left(k_{2} \bar{u}_{1}+\bar{u}_{2}\right)^{2}}\right\|_{1} \\
& \quad=\left\|\frac{1}{\left(k_{2} u_{1}+u_{2}\right)\left(k_{2} \bar{u}_{1}+\bar{u}_{2}\right)^{2}}\left(\bar{u}_{1} \bar{u}_{2} w_{1}+\bar{u}_{1}^{2} w_{2}\right)\right\|_{1} \\
& \leq \\
& \quad\left\{\left\|\frac{1}{k_{2} \bar{u}_{1}+\bar{u}_{2}}\right\|_{1}\left\|\frac{\bar{u}_{1} \bar{u}_{2}}{\left(k_{2} u_{1}+u_{2}\right)\left(k_{2} \bar{u}_{1}+\bar{u}_{2}\right)}\right\|_{1}\right. \\
& \left.\quad+\left\|\frac{1}{k_{2} u_{1}+u_{2}}\right\|_{1}\left\|\frac{\bar{u}_{1}^{2}}{\left(k_{2} \bar{u}_{1}+\bar{u}_{2}\right)^{2}}\right\|_{1}\right\}\left(\left\|w_{1}\right\|_{1}+\left\|w_{2}\right\|_{1}\right) .
\end{aligned}
$$

Suppose that

$$
\left\|w_{1}\right\|_{1}+\left\|w_{2}\right\|_{1}<\frac{1}{2} \inf _{x \in G} \min \left\{\bar{u}_{1}(x), \bar{u}_{2}(x)\right\}=: \delta .
$$

Then we find that

$$
\begin{aligned}
u_{j}(x) & =u_{j}(x)-\bar{u}_{j}(x)+\bar{u}_{j}(x) \\
& =\bar{u}_{j}(x)-w_{j}(x) \\
& \geq \inf _{x \in G} \bar{u}_{j}(x)-\left\|w_{j}\right\|_{1} \\
& \geq \frac{1}{2} \inf _{x \in G} \bar{u}_{j}(x) \\
& \geq \frac{1}{2} \inf _{x \in G} \min _{j=1,2}\left\{\bar{u}_{j}(x)\right\} \\
& =: c_{1}
\end{aligned}
$$

for all $x \in G$. Hence when (19) holds, we see that

$$
k_{2} u_{1}(x)+u_{2}(x) \geq\left(k_{2}+1\right) c_{1} \quad(x \in G)
$$

which implies that

$$
\left\|\frac{1}{k_{2} u_{1}+u_{2}}\right\|_{1} \leq \frac{1}{\left(k_{2}+1\right) c_{1}}=: C_{1} .
$$

Combining (18) and (20) we find that for $\|w\|_{2}=\left\|w_{1}\right\|_{1}+\left\|w_{2}\right\|_{1}<\delta$

$$
\begin{aligned}
&\left\|\frac{\bar{u}_{1} u_{1}}{\left(k_{2} u_{1}+u_{2}\right)\left(k_{2} \bar{u}_{1}+\bar{u}_{2}\right)}-\frac{\bar{u}_{1}^{2}}{\left(k_{2} \bar{u}_{1}+\bar{u}_{2}\right)^{2}}\right\|_{1} \\
& \leq\left\{C_{1}\left\|\frac{1}{k_{2} \bar{u}_{1}+\bar{u}_{2}}\right\|_{1}\left\|\frac{\bar{u}_{1} \bar{u}_{2}}{k_{2} \bar{u}_{1}+\bar{u}_{2}}\right\|_{1}+C_{1}\left\|\frac{\bar{u}_{1}^{2}}{\left(k_{2} \bar{u}_{1}+\bar{u}_{2}\right)^{2}}\right\|_{1}\right\} \\
& \times\left(\left\|w_{1}\right\|_{1}+\left\|w_{2}\right\|_{1}\right) \\
&=: C_{2}\|w\|_{2}
\end{aligned}
$$

where $C_{2}$ does not depend on $w$. Similarly we find that

$$
\left\|\frac{\bar{u}_{2} u_{2}}{\left(k_{2} u_{1}+u_{2}\right)\left(k_{2} \bar{u}_{1}+\bar{u}_{2}\right)}-\frac{\bar{u}_{2}^{2}}{\left(k_{2} \bar{u}_{1}+\bar{u}_{2}\right)^{2}}\right\|_{1} \leq C_{3}\|w\|_{2}
$$

for $\|w\|_{2}<\delta$. Hence we find from (17) that

$$
f(\bar{u}+w)-f(\bar{u})-\left(a_{1} w_{1}+a_{2} w_{2}\right)=g(w)
$$

where $\|g(w)\|_{1} \leq C_{4}\|w\|_{2}^{2}$ for $\|w\|_{2}<\delta$ which completes the proof 
Remark 2. Consequently, the above considerations show that the mapping $f$ : $C(\bar{G}) \times C(\bar{G}) \mapsto C(\bar{G})$ is differentiable in the neighbourhood of $\bar{u}$.

Denote $U=\left(U_{1}, U_{2}\right)=u-\bar{u}$. Substracting equations (5) - (6) and (8) - (9) side by side we get

$$
\left.\begin{array}{l}
\frac{\partial U_{1}}{\partial t}=f(U+\bar{u})-f(\bar{u})-k_{d} U_{1} \\
\frac{\partial U_{2}}{\partial t}=D \frac{\partial^{2} U_{2}}{\partial x^{2}}-c(t) \frac{\partial\left(U_{2}+\bar{u}_{2}\right)}{\partial x}+\bar{c} \frac{\partial \bar{u}_{2}}{\partial x}-k_{1} f(\bar{u}+U)+k_{1} f(\bar{u})
\end{array}\right\}
$$

for $(x, t) \in G \times \mathbb{R}_{+}$,

$$
\left.\begin{array}{l}
\left.\frac{\partial U_{2}}{\partial x}(0, t)=\frac{c(t)}{D}\left[\left(U_{2}+\bar{u}_{2}\right)(0, t)-S(t)\right]-\frac{\bar{c}}{D}\left[\bar{u}_{2}(0)-\bar{S}\right)\right] \\
\frac{\partial U_{2}}{\partial x}(1, t)=0
\end{array}\right\}
$$

for $t \in \mathbb{R}_{+}$, and

$$
\left.\begin{array}{l}
U_{1}(x, 0)=u_{10}(x)-\bar{u}_{1}(x) \\
U_{2}(x, 0)=u_{20}(x)-\bar{u}_{2}(x)
\end{array}\right\}
$$

for $x \in G$. Due to Lemma 1 problem (23) - (25) can be put into the form

$$
\left.\begin{array}{l}
\frac{\partial U_{1}}{\partial t}=\left(a_{1}-k_{d}\right) U_{1}+a_{2} U_{2}+g(U) \\
\frac{\partial U_{2}}{\partial t}=D \frac{\partial^{2} U_{2}}{\partial x^{2}}-c(t) \frac{\partial U_{2}}{\partial x}-[c(t)-\bar{c}] \frac{\partial \bar{u}_{2}}{\partial x}-k_{1} a_{1} U_{1}-k_{1} a_{2} U_{2}-k_{1} g(U)
\end{array}\right\}
$$

for $(x, t) \in G \times \mathbb{R}_{+}$,

$$
\left.\begin{array}{l}
\frac{\partial U_{2}}{\partial x}(0, t)=\frac{c(t)}{D} U_{2}(0, t)+\frac{c(t)-\bar{c}}{D} \bar{u}_{2}(0)-\frac{c(t)}{D} S(t)+\frac{\bar{c}}{D} \bar{S} \\
\frac{\partial U_{2}}{\partial x}(1, t)=0
\end{array}\right\}
$$

for $t \in \mathbb{R}_{+}$,

$$
\left.\begin{array}{l}
U_{1}(x, 0)=u_{10}(x)-\bar{u}_{1}(x) \\
U_{2}(x, 0)=u_{20}(x)-\bar{u}_{2}(x) .
\end{array}\right\}
$$

By substituting the transformed variable $v=\left(v_{1}, v_{2}\right)=\left(\kappa U_{1}, U_{2}+s(t)\right)$ with $\kappa=$ $\left(k_{1} \frac{a_{1}}{a_{2}}\right)^{\frac{1}{2}}$ into system (26) - (28) and by making the definition

$$
s(t)=\frac{D}{c(t)}\left[\frac{c(t)-\bar{c}}{D} \bar{u}_{2}(0)-\frac{c(t)}{D} S(t)+\frac{\bar{c}}{D} \bar{S}\right]
$$


we obtain the system

$$
\left.\begin{array}{rl}
\frac{\partial v_{1}}{\partial t}= & \left(a_{1}-k_{d}\right) v_{1}+\kappa a_{2} v_{2}-\kappa a_{2} s(t)+\kappa g\left(\frac{1}{\kappa} v_{1}, v_{2}-s(t)\right) \\
\frac{\partial v_{2}}{\partial t}= & D \frac{\partial^{2} v_{2}}{\partial x^{2}}-c(t) \frac{\partial v_{2}}{\partial x}-(c(t)-\bar{c}) \frac{\partial \bar{u}_{2}}{\partial x}-\frac{k_{1} a_{1}}{\kappa} v_{1} \\
& -k_{1} a_{2} v_{2}+k_{1} a_{2} s(t)-k_{1} g\left(\frac{1}{\kappa} v_{1}, v_{2}-s(t)\right)+\dot{s}(t)
\end{array}\right\}
$$

for $(x, t) \in G \times \mathbb{R}_{+}$,

$$
\left.\begin{array}{l}
\frac{\partial v_{2}}{\partial x}(0, t)=\frac{c(t)}{D} v(0) \\
\frac{\partial v_{2}}{\partial x}(1, t)=0
\end{array}\right\}
$$

for $t \in \mathbb{R}_{+}$, and

$$
\left.\begin{array}{l}
v_{1}(x, 0)=\kappa\left(u_{10}(x)-\bar{u}_{1}(x)\right) \\
v_{2}(x, 0)=u_{20}(x)-\bar{u}_{2}(x)+s(0) .
\end{array}\right\}
$$

Define a linear operator

$$
A(t): C(\bar{G}) \times L_{p}(G) \mapsto C(\bar{G}) \times L_{p}(G)
$$

by

$$
\begin{aligned}
D(A(t)) & =C(\bar{G}) \times\left\{w_{2} \in W^{2, p}(G) \mid\left(\frac{\partial w_{2}}{\partial x}-\frac{c(t)}{D} w_{2}\right)(0)=0, \frac{\partial w_{2}}{\partial x}(1)=0\right\} \\
A(t) w & =-\left(\left(a_{1}-k_{d}\right) w_{1}+\kappa a_{2} w_{2}, D \frac{\partial^{2} w_{2}}{\partial x^{2}}-c(t) \frac{\partial w_{2}}{\partial x}-\frac{k_{1} a_{1}}{\kappa} w_{1}-k_{1} a_{2} w_{2}\right)
\end{aligned}
$$

for $w \in D(A(t))$. Since $W^{2, p}(G) \subset C^{1}(\bar{G})$ (because $\left.n=1\right)$ for $p>1$ the terms $w_{2}(0)$, $w_{2}(1)$, and $\frac{\partial w_{2}}{\partial x}(0)$ are well-defined. Furthermore, define

$$
\begin{aligned}
F(t, w)= & \left(-\kappa a_{2} s(t)+\kappa g\left(\frac{1}{\kappa} w_{1}, w_{2}-s(t)\right)\right. \\
& \left.-[c(t)-\bar{c}] \frac{\partial \bar{u}_{2}}{\partial x}+k_{1} a_{2} s(t)-k_{1} g\left(\frac{1}{\kappa} w_{1}, w_{2}-s(t)\right)+\dot{s}(t)\right) .
\end{aligned}
$$

Then problem (28) - (32) gets the abstract form

$$
\left.\begin{array}{rl}
\frac{d v}{d t}+A(t) v & =F(t, v) \\
v(0) & =v_{0}
\end{array}\right\}
$$

where

$$
v_{0}(x)=\left(\kappa\left[u_{10}(x)-\bar{u}_{1}(x)\right], u_{20}(x)-\bar{u}_{2}(x)+s(0)\right) .
$$




\section{Stability of solutions}

3.1 The evolution operator of $\boldsymbol{A}(\boldsymbol{t})$. We use the notations and theory of [2]. Let for $s \in[-1,1]$ and $p \in(1, \infty)$ be

$$
W_{B}^{s, p}(G)= \begin{cases}W^{s, p}(G) & \text { if } 0 \leq s \leq 1 \\ \left(W^{-s, p^{\prime}}(G)\right)^{\prime} & \text { if }-1 \leq s \leq 0 .\end{cases}
$$

Define time-dependent Sobolev spaces $W_{B(\ell)}^{2, p}(G)$ by

$$
W_{B(t)}^{2, p}(G)=\left\{w \in W^{2, p}(G) \mid D \frac{\partial w}{\partial x}(0)-c(t) w(0)=0 \text { and } \frac{\partial w}{\partial x}(1)=0\right\} .
$$

In addition we define Banach spaces

$$
E(t)=C(\bar{G}) \times W_{B(t)}^{2, p}(G) \quad \text { and } \quad E_{\alpha+\frac{1}{2}}=C(\bar{G}) \times W_{B}^{2 \alpha, p}(G)
$$

where $-\frac{1}{2} \leq \alpha \leq \frac{1}{2}$. The operator $A(t)$ can be interpreted as a bounded operator $E_{1} \mapsto E_{0}$ (that is, $A(t) \in L\left(E_{1}, E_{0}\right)$ ) by

$$
A(t)=\left(\begin{array}{cc}
A_{11} & A_{12} \\
A_{21} & A_{22}(t)
\end{array}\right)
$$

where for $w=\left(w_{1}, w_{2}\right) \in E_{1}$

$$
A_{11} w_{1}=\left(k_{d}-a_{1}\right) w_{1}, \quad A_{12} w_{2}=-\kappa a_{2} w_{2}, \quad A_{21} w_{1}=\frac{k_{1} a_{1}}{\kappa} w_{1}
$$

and where

$$
A_{22}(t): W^{1, p}(G) \mapsto W_{B}^{-1, p}(G)=\left(W^{1, p^{\prime}}(G)\right)^{\prime}
$$

is the opcrator corresponding to the antilinear form $a_{22}(t): W^{1, p}(G) \times W^{1, p^{\prime}}(G) \mapsto C$ such that

$$
a_{22}(t)\left(w_{2}, v_{2}\right)=\int_{0}^{1}\left(D \frac{\partial w_{2}}{\partial x} \frac{\overline{\partial v_{2}}}{\partial x}+k_{1} a_{2} w_{2} \bar{v}_{2}+c(t) \frac{\partial w_{2}}{\partial x} \bar{v}_{2}\right) d x+c(t) w_{2}(0) \bar{v}_{2}(0)
$$

that is,

$$
\left\langle A_{22}(t) w_{2}, v_{2}\right\rangle:=\int_{0}^{1}\left(-D \frac{\partial^{2} w_{2}}{\partial x^{2}}+c(t) \frac{\partial w_{2}}{\partial x}+k_{1} a_{2} w_{2}\right) \bar{v}_{2} d x=a_{22}(t)\left(w_{2}, v_{2}\right)
$$

(which follows by partial integration).

For the definition of the (parabolic) evolution operator

$$
U(t, \tau): C(\bar{G}) \times L_{p}(G) \mapsto C(\bar{G}) \times L_{p}(G)
$$

for $A(t)$ we refer to [2: pp. $45-47]$. The solution $v$ of problem (33) satisfies the (Volterra) integral equation

$$
v(t)=U(t, 0) v_{0}+\int_{0}^{t} U(t, \tau) F(\tau, v(\tau)) d \tau .
$$

For this operator the following estimates are proved. 
Theorem 1. There exists a constant $\delta>0$ such that when $\left[\left.c\right|_{C^{--}\left(\overline{\mathbb{R}}_{+}\right)}<\delta\right.$, where $0<\rho \leq 1$, then the operator $A(t)$ has an evolution operator $U(t, \tau): C(\bar{G}) \times L_{p}(G) \mapsto$ $C(\bar{G}) \times L_{p}(G)$ and there exist $\alpha \in\left(\frac{1}{2}, 1\right]$ and $\beta>0$ such that for $w \in C(\bar{G}) \times W^{\alpha, p}(G)$ and for $0<\tau<t$

$$
\begin{aligned}
& \|U(t, \tau) w\|_{3} \leq M e^{-\beta(t-\tau)}\|w\|_{3} \\
& \|U(t, \tau) w\|_{3} \leq M(t-\tau)^{-\left(\alpha-\frac{1}{2}\right)} e^{-\beta(t-\tau)}\|w\|_{4}
\end{aligned}
$$

where we use the abbreviations $\|\cdot\|_{3}=\|\cdot\|_{C(\bar{G}) \times W^{a, p}(G)}$ and $\|\cdot\|_{4}=\|\cdot\|_{C(\bar{G}) \times L_{p}(G)}$.

For the definition of the quantity $[\cdot]_{C^{-}-\left(\overline{\mathbb{R}}_{+}\right)}$see [2: p. 40].

Proof of Theorem 1. We shall only outline the proof and omit many details. The proof is given in Parts A, B and C.

Part A: Let $A_{0}(t)=A(t)_{\mid E(t)}$. It is well-known that the operator $A_{0}(t) \quad(t \geq 0)$ generates an analytic semigroup on $E_{\frac{1}{2}}$ with domain $E(t)$ (sec, e.g., $\left.[1,9,10,18,21]\right)$ and then with the notations of [2] $A_{0}(t) \in H\left(E(t), E_{\frac{1}{2}}\right)$.

Part B: Using the relation $\kappa=\left(k_{1} \frac{a_{1}}{a_{2}}\right)^{\frac{1}{2}}$ and the fact that, for $w \in L_{2}(G) \times$ $W_{B(t)}^{2,2}(G)$

$$
D \frac{\partial w_{2}}{\partial x}(0) \bar{w}_{2}(0)=-c(t) w_{2}(0) \bar{w}_{2}(0) \quad \text { and } \quad \cdot \frac{\partial w_{2}}{\partial x}(1)=0
$$

we find that, for all $w \in L_{2}(G) \times W_{B(t)}^{2,2}(G)$,

$$
\begin{aligned}
\Re(A(t) w, w\rangle_{L_{2}(G)^{2}} & \Re\left[\left(k_{d}-a_{1}\right) \int_{0}^{1} w_{1} \bar{w}_{1} d x+k_{1} a_{2} \int_{0}^{1} w_{2} \bar{w}_{2} d x\right. \\
= & -\kappa a_{2} \int_{0}^{1} w_{2} \bar{w}_{1} d x+\frac{k_{1} a_{1}}{\kappa} \int_{0}^{1} w_{1} \bar{w}_{2} d x \\
& \left.+c(t) w_{2}(0) \bar{w}_{2}(0)+D \int_{0}^{1} \frac{\partial w_{2}}{\partial x} \frac{\overline{\partial w_{2}}}{\partial x} d x+c(t) \int_{0}^{1} \frac{\partial w_{2}}{\partial x} \bar{w}_{2} d x\right] \\
= & \left(k_{d}-a_{1}\right) \int_{0}^{1}\left|w_{1}\right|^{2} d x+k_{1} a_{2} \int_{0}^{1}\left|w_{2}\right|^{2} d x \\
& -\kappa a_{2} \Re \int_{0}^{1} w_{2} \bar{w}_{1} d x+\frac{k_{1} a_{1}}{\kappa} \Re \int_{0}^{1} w_{1} \bar{w}_{2} d x \\
& +c(t)\left|w_{2}(0)\right|^{2}+D \int_{0}^{1} \frac{\partial w_{2}}{\partial x} \frac{\partial w_{2}}{\partial x} d x+\left.c(t)\right|_{0} ^{1} \frac{1}{2}\left|w_{2}\right|^{2} \\
= & \left(k_{d}-a_{1}\right) \int_{0}^{1}\left|w_{1}\right|^{2} d x+k_{1} a_{2} \int_{0}^{1}\left|w_{2}\right|^{2} d x \\
& -D \int_{0}^{1}\left|\frac{\partial w_{2}}{\partial x}\right|^{2} d x+\frac{c(t)}{2}\left[\left|w_{2}(1)\right|^{2}+\left|w_{2}(0)\right|^{2}\right] .
\end{aligned}
$$


Equation (37) immediately gives that for $w \in L_{2}(G) \times W_{B(t)}^{2,2}(G)$ we have the estimate

$$
\Re\langle A(t) w, w\rangle_{\left.L_{2}(G)\right)^{2}} \geq \min \left\{k_{d}-a_{1}, k_{1} a_{2}\right\}\|w\|_{L_{2}(G) \times W^{1,2}(G)}^{2} .
$$

Part C: Equation (38) and Part A imply that for $p=2$

$$
\sigma\left(A_{0}(t)\right) \subset\{z \in C \mid \Re z \geq \gamma\} \quad(t \geq 0)
$$

where $\gamma=\min \left\{k_{d}-a_{1}, k_{1} a_{2}\right\}$. Here $\gamma$ is positive since

$$
k_{d}-a_{1}=\frac{k_{d}\left(\mu_{m}-k_{d}\right)}{\mu_{m}} \quad \text { and } \quad k_{1} a_{2}=k_{1} \frac{\left(\mu_{m}-k_{d}\right)^{2}}{\mu_{m} k_{2}}
$$

are positive for relevant parameter values. Since $G$ is bounded we know (from elliptic theory) that inclusion (39) is valid for any $p>1$.

The continuity of $c$, inclusion (39) and well-known estimates for elliptic operators (cf. [1: p. 42]) imply that for any $\sigma>-\gamma$ and $\omega>0$

$$
\sigma I+A(\cdot) \in C\left(\overline{\mathbb{R}}_{+}, H\left(E_{1}, E_{0}, \kappa, \omega\right)\right)
$$

where $\kappa>0$. The space $C\left(\overline{\mathbb{R}}_{+}, H\left(E_{1}, E_{0}, \kappa, \omega\right)\right)$ is defined as in [2: p. 11]. Furthermore, we find that (for $0<\rho \leq 1$ )

$$
[A(\cdot)]_{C^{\bullet-}\left(\overline{\mathbb{R}}_{+}, L\left(E_{1}, E_{0}\right)\right)} \leq[c]_{C^{p-\left(\overline{\mathbb{R}}_{+}\right)}} .
$$

Hence when $[c]_{C^{\rho-(}\left(\overline{\mathbb{R}}_{+}\right)}$is small enough we find that [2: Assumption (II. 5.0.1)] holds. Choosing $\beta=\beta_{-}=\frac{1}{2},[2$ : Lemma II.5.1.3] implies the assertion

Remark 3. Based on the assumption $\|\dot{c}\|_{C\left(\overline{\mathbb{R}}_{+}\right)}<\delta$ it can be concluded that $[c]_{C \rho-\left(\overline{\mathbb{R}}_{+}\right)}<\delta$ when $\rho=1$.

The evolution operator of $A(t)-\beta_{1} I$ is

$$
\mathbf{U}(t, \tau)=e^{t \beta_{1} I} \circ U(t, \tau) \circ e^{-\tau \beta_{1} I}
$$

for $0<\beta_{1}<\beta$. This evolution operator satisfies for $w \in C(\bar{G}) \times W^{\alpha, p}(G)$ and for $0<\tau<t$ the estimates

$$
\begin{aligned}
\|\mathbf{U}(t, \tau) w\|_{3} & \leq M e^{-\left(\beta-\beta_{1}\right)(t-\tau)}\|w\|_{3} \\
\|\mathbf{U}(t, \tau) w\|_{3} & \leq M(t-\tau)^{-\left(\alpha-\frac{1}{2}\right)} e^{-\left(\beta-\beta_{1}\right)(t-\tau)}\|w\|_{4}
\end{aligned}
$$

where the norms are given in Theorem 1.

3.2. Local asymptotic stability. Here we prove a result about the long-time behaviour for the solutions of problem (5) - (7). The basic idea of the proof is standard (cf., e.g., [3]). However, the verification contains some special estimates which must be computed. In the proof we establish the required estimates. The proof is given in Parts $A, B$, and $C$. 

if

Theorem 2. Let $\max \left\{\frac{1}{p}, \frac{1}{2}\right\}<\alpha \leq 1$. Then there exists a constant $\delta>0$ such that

$$
\begin{aligned}
\left\|e^{\beta_{1}(\cdot)}(c-\bar{c})\right\|_{\infty}<\delta, & \left\|e^{\beta_{1}(\cdot)} \dot{c}\right\|_{\infty}<\delta \\
\left\|e^{\beta_{1}(\cdot)}(S-\bar{S})\right\|_{\infty}<\delta, & \left\|e^{\beta_{1}(\cdot)} \dot{S}\right\|_{\infty}<\delta
\end{aligned}
$$

and if

$$
\left\|u_{0}-\bar{u}\right\|_{C(\bar{G}) \times W^{a, p}(G)}<\delta
$$

then

$$
\lim _{t \rightarrow \infty}\|u(\cdot, t)-\bar{u}\|_{C(\bar{G}) \times W^{\alpha, p}(G)}=0 .
$$

Proof. Part A: Denote $V=e^{\beta_{1} t} v$. Then system (33) becomes

$$
\left.\begin{array}{rl}
\frac{d V}{d t}+\left[A(t)-\beta_{1} I\right] V & =e^{\beta_{1} t} F\left(t, e^{-\beta_{1} t} V\right) \\
V(0) & =e^{\beta_{1} 0} v_{0}=: V_{0}
\end{array}\right\}
$$

Consider the nonlinearity $\bar{F}(t, V)=e^{\beta_{1} t} F\left(t, e^{-\beta_{1} t} V\right)$. We find that

$$
\begin{aligned}
\bar{F}(t, V) & =e^{\beta_{1} t}\left(-\kappa a_{2} s(t)+\kappa g,-[c(t)-\bar{c}] \frac{\partial \bar{u}_{2}}{\partial x}+k_{1} a_{2} s(t)-k_{1} g+\dot{s}(t)\right) \\
g & =g\left(\frac{1}{\kappa} e^{-\beta_{1} t} V_{1}, e^{-\beta_{1} t} V_{2}-s(t)\right) .
\end{aligned}
$$

Since $G=(0,1) \subset \mathbb{R}$, the Sobolev inequality implies that

$$
\|u\|_{C(\bar{G})} \leq C_{1}\|u\|_{W^{a, p}(G)} \quad\left(u \in W^{\alpha, p}(G)\right)
$$

when $\frac{1}{p}<\alpha$. Hence due to Lemma 1 we find that there exists constants $0<\delta^{\prime}<1$ and $C_{2}>0$ such that for $\|V\|_{3}+\|s\|_{\infty}<\delta^{\prime}$ (c.f. Theorem 1 for the norms)

$$
\|\bar{F}(t, V)\|_{4} \leq C_{2}\left\{\|V\|_{3}^{2}+\left|e^{\beta_{1} t} s(t)\right|+\left|e^{\beta_{1} t}[c(t)-\bar{c}]\right|+\left|e^{\beta_{1} t} \dot{s}(t)\right|\right\} .
$$

Part B: We find, by adding and substracting a term in (29), that

$$
s(t)=\frac{D}{c(t)}\left\{\frac{c(t)-\bar{c}}{D} \bar{u}_{2}(0)-\frac{c(t)}{D}[S(t)-\bar{S}]-\frac{\bar{S}}{D}[c(t)-\bar{c}]\right\} .
$$

By differentiating this equality we have

$$
\begin{aligned}
\dot{s}(t)= & -\frac{D \dot{c}(t)}{c(t)^{2}}\left\{\frac{c(t)-\bar{c}}{D} \bar{u}_{2}(0)-\frac{c(t)}{D}[S(t)-\bar{S}]-\frac{\bar{S}}{D}[c(t)-\bar{c}]\right\} \\
& +\frac{D}{c(t)}\left\{\frac{\dot{c}(t)}{D} \bar{u}_{2}(0)-\frac{\dot{c}(t)}{D} S(t)-\frac{c(t)}{D} \dot{S}(t)-\frac{\bar{S}}{D} \dot{c}(t)\right\} .
\end{aligned}
$$


The assumptions imply that $|c|,|\dot{c}|$ and $|S|,|\dot{S}|$ are bounded from above and that $c$ is bounded from below by a positive constant. Hence there exist constants $C_{3}>0$ and $C_{4}>0$ such that

$$
\begin{aligned}
& |s(t)| \leq C_{3}\{|c(t)-\bar{c}|+|S(t)-\bar{S}|\} \\
& |\dot{s}(t)| \leq C_{4}\{|c(t)-\bar{c}|+|S(t)-\bar{S}|+|\dot{c}(t)|+|\dot{S}(t)|\} .
\end{aligned}
$$

In addition, we find that $\left\|V_{0}\right\|_{3}<\delta^{\prime}$ if

$$
\left\|u_{0}-\bar{u}\right\|_{3}<\frac{\delta^{\prime}}{2 \max \{\kappa, 1\}} \quad \text { and } \quad|s(0)|<\frac{\delta^{\prime}}{2} .
$$

Part C: Suppose that $\left\|V_{0}\right\|_{3}+\|s\|_{\infty}<\delta^{\prime}$. Then there exists $t^{\prime}>0$ such that $\|V(t)\|_{3}+\|s\|_{\infty}<\delta^{\prime}$ for $t \in\left(0, t^{\prime}\right)$. Due to (47) and (50) - (52) we get for $t \in\left(0, t^{\prime}\right)$

$$
\begin{aligned}
\|V(t)\|_{3}= & \left\|\mathrm{U}(t, 0) V_{0}+\int_{0}^{t} \mathrm{U}(t, \tau) \bar{F}(\tau, V(\tau)) d \tau\right\|_{3} \\
\leq & \left\|\mathrm{U}(t, 0) V_{0}\right\|_{3}+\int_{0}^{t}\|\mathrm{U}(t, \tau) \bar{F}(\tau, V(\tau))\|_{3} d \tau \\
\leq & M e^{-\left(\beta-\beta_{1}\right) t}\left\|V_{0}\right\|_{3}+\int_{0}^{t} M(t-\tau)^{-\left(\alpha-\frac{1}{2}\right)} e^{-\left(\beta-\beta_{1}\right)(t-\tau)}\|\bar{F}\|_{4} d \tau \\
\leq & M e^{-\left(\beta-\beta_{1}\right) t}\left\|V_{0}\right\|_{3}+\int_{0}^{t} M(t-\tau)^{-\left(\alpha-\frac{1}{2}\right)} e^{-\left(\beta-\beta_{1}\right)(t-\tau)} \\
& \times C_{2}\left\{\|V(\tau)\|_{3}^{2}+\left|e^{\beta_{1} \tau} s(\tau)\right|+\left|e^{\beta_{1} \tau}[c(\tau)-\bar{c}]\right|+\left|e^{\beta_{1} \tau} \dot{s}(\tau)\right|\right\} d \tau \\
\leq & M e^{-\left(\beta-\beta_{1}\right) t}\left\|V_{0}\right\|_{3}+M C_{2}\left\{\sup _{t \in\left[0, t^{\prime}\right)}\|V(\tau)\|_{3}^{2}+\left\|e^{\beta_{1}(\cdot)} s\right\|_{\infty}\right. \\
& \left.+\left\|e^{\beta_{1}(\cdot)}(c-\bar{c})\right\|_{\infty}+\left\|e^{\beta(\cdot)} \dot{s}\right\|_{\infty}\right\} \int_{0}^{\infty} \tau \tau^{-\left(\alpha-\frac{1}{2}\right)} e^{-\left(\beta-\beta_{1}\right) \tau} d \tau
\end{aligned}
$$

where the abbreviation $\|\bar{F}\|_{4}=\|\bar{F}(\tau, V(\tau))\|_{4}$ was used. From (50) - (53) we are able to conclude that there exists $\delta>0$ such that if

$$
\begin{aligned}
\left\|e^{\beta_{1}(\cdot)}(c-\bar{c})\right\|_{\infty}<\delta, & \left\|e^{\beta_{1}(\cdot)} \dot{c}\right\|_{\infty}<\delta \\
\left\|e^{\beta_{1}(\cdot)}(S-\bar{S})\right\|_{\infty}<\delta, & \left\|e^{\beta_{1}(\cdot)} \dot{S}\right\|_{\infty}<\delta
\end{aligned}
$$

and if

$$
\left\|u_{0}-\bar{u}\right\|_{3}<\delta
$$

then

$$
\|V(t)\|_{3} \leq \delta_{1} \quad\left(t \in\left[0, t_{1}\right)\right) \quad \Longrightarrow \quad\|V(t)\|_{3} \leq \delta_{0}<\delta_{1} \quad\left(t \in\left[0, t_{1}\right)\right) .
$$

This implies that $V$ is bounded $\left(\|V(t)\|_{3} \leq \delta_{1}\right)$ on $\overline{\mathbb{R}}_{+}$, that is, $v$ satisfies $\|v(t)\|_{3} \leq$ $C_{5} e^{-\beta_{1} t}$ for all $t \in \overline{\mathbb{R}}$. Hence, due to the assumption, we have

$$
\|u(t)-\bar{u}\|_{3}=\|U(t)\|_{3}=\left\|\left(\frac{1}{\kappa} v_{1}, v_{2}-s(t)\right)\right\|_{3} \leq C_{6}\left\{\|v(t)\|_{3}+|s(t)|\right\} \leq C_{7} e^{-\beta_{1} t} .
$$

This completes the proof 


\section{A remark on the output tracking problem}

One of the specific questions in the controller design related to problem (5) - (7) is the solvability of the following output tracking problem:

For the given reference function $y^{*} \in C\left(\overline{\mathbb{R}}_{+}\right)$with $0<y^{*}(t)<S(t)$ for all $t \in \overline{\mathbb{R}}_{+}$ find the control $c=c\left(u, y^{*}\right)$ (sufficiently smooth) such that the output

$$
y(t):=u_{2}(1, t)=y^{*}(t) \quad\left(t \in \overline{\mathbb{R}}_{+}\right)
$$

at least approximately and/or asymptotically. Also, the stability (in appropriate spaces) of the associated closed loop system is significant in practical situations. For example, changes in $S$ cause disturbances in the state and output of the system.

In mathematical setting the producing of the prescribed reference output $y^{*}(t)$ means the non-homogeneous boundary condition

$$
u_{2}(1, t)=y^{*}(t)
$$

From

$$
\left.\begin{array}{rl}
\frac{\partial u_{1}}{\partial t} & =-k_{d} u_{1}+f\left(u_{1}, u_{2}\right) \\
u_{1}(x, 0) & =\bar{u}_{1}(x)
\end{array}\right\}
$$

we can at least in theory solve the unknown $u_{1}$, say $u_{1}=\theta\left(u_{2}, t\right)$. From (6) we obtain

$$
c(t)=\frac{D \frac{\partial u_{2}}{\partial x}(0, t)}{u_{2}(0, t)-s(t)} .
$$

Hence we find that $u_{2}$ satisfies the problem

$$
\begin{aligned}
\frac{\partial u_{2}}{\partial t} & =D \frac{\partial^{2} u_{2}}{\partial x^{2}}-\frac{D \frac{\partial u_{2}}{\partial x}(0, t)}{u_{2}(0, t)-s(t)} \frac{\partial u_{2}}{\partial x}-k_{1} f\left(\theta\left(u_{2}, t\right), u_{2}\right) \\
u_{2}(1, t) & =y^{*}(t), \quad \frac{\partial u_{2}}{\partial x}(1, t)=0 \\
u_{2}(x, 0) & =\bar{u}_{2}(x) .
\end{aligned}
$$

Denoting $V_{1}=u_{2}$ and $V_{2}=\frac{\partial u_{2}}{\partial x}$ problem (55) - (57) gets the form

$$
\begin{aligned}
& \frac{\partial V_{1}}{\partial x}=V_{2} \\
& \frac{\partial V_{2}}{\partial x}=\frac{1}{D} \frac{\partial V_{1}}{\partial t}+\frac{V_{2}(0, t)}{V_{1}(0, t)-s(t)} V_{2}+\frac{k_{1}}{D} f\left(\theta\left(V_{1}, t\right), V_{1}\right) \\
& V_{1}(x, 0)=\bar{u}_{2}(x) \\
& V_{1}(1, t)=y^{*}(t), \quad V_{2}(1, t)=0 \text {. }
\end{aligned}
$$


Furthermore, we denote $W_{1}=V_{1}-\bar{u}_{2}(x)$ and $W_{2}=V_{2}$. Then we have for $W=\left(W_{1}, W_{2}\right)$

$$
\left.\begin{array}{rl}
\frac{\partial W}{\partial x}+A W & =F(t, W) \\
W(1) & =\left(y^{*}(t)-\bar{u}_{2}(1), 0\right)
\end{array}\right\}
$$

where $A$ is an operator $L_{p}\left(\mathbb{R}_{+}\right)^{2} \mapsto L_{p}\left(\mathbb{R}_{+}\right)^{2}$ such that

$$
D(A)=L_{p}\left(\mathbb{R}_{+}\right) \times W_{0}^{p, 1}\left(\mathbb{R}_{+}\right), \quad A W=-\left(W_{2}, \frac{1}{D} \frac{\partial W_{1}}{\partial t}\right)
$$

and

$$
F(t, W)=\left(-\frac{\partial \bar{u}_{2}}{\partial x}, \frac{W_{2}(0)}{W_{1}(0)+\bar{u}_{2}(0)-s(t)} W_{2}+\frac{k_{1}}{D} f\left(\theta\left(W_{1}+\bar{u}_{2}, t\right), W_{1}+\bar{u}_{2}\right)\right) .
$$

In this approach the existence and stability properties of system (61) would be interesting. The corresponding control law is given by

$$
C=\frac{\dot{W}_{2}(0, t)}{W_{1}(0, t)+\bar{u}_{2}(0)-s(t)}
$$

The solvability of the output tracking problem given above remains open.

Acknowledgement. Part of this work was supported by the Academy of Finland, Research Council for Natural Sciences and Engineering (Project no: 37462, 1997) which is greatly acknowledged.

\section{References}

[1] Amann, H.: Nonhomogeneous linear and quasilinear elliptic and parabolic boundary value problems. In: Function Spaces, Differential Operators and Nonlinear Analysis (eds.: H.J. Schmeisser and H. Tricbel; Teubner-Texte zur Math.: Vol. 133). Stuttgart-Leipzig: Teubner 1993, pp. 9 - 126.

[2] Amann, H.: Linear and Quasilinear Parabolic Problems. Vol. I. Basel: Birkhäuser 1995.

[3] Banks, S. P.: State-Space and Frequency-Domain Methods in the Control of Distributed Parameter Systems. London: Peter Peregrinus Ltd. 1983.

[4] Curtain, R. F.: Equivalence of input-output stability and exponential slability for infinitedimensional systems. Math. Systems Theory 21 (1988), $19-48$.

[5] Curtain, R. F. and H. J. Zwart: An Introduction to Infinite-Dimensional Linear Systems Theory (Texts in Applied Mathematics). Berlin: Springer 1995.

[6] Danckwerts, P. V.: Continuous flow systems. Chem. Eng. Sci. 2 (1953), 1 - 13.

[7] Dochain, D., Babary, J. P. and N. Tali-Maamar: Modelling and adaptive control of nonlinear distributed parameter bioreactors via orthogonal collocation. Automatica 28 (1992), $273-283$. 
[8] Dochain, D.: Contribution to the analysis and control of distributed parameter systems with application to (bio)chemical processes and robotics. Thesis. Louvain (Belgium): Universite Catholique 1994.

[9] Friedman, A.: Partial Differential Equations. New York: Holt, Rinehart \& Winston 1969.

[10] Goldstein, J. A.: Semigroups of Linear Operators and Applications. Oxford: Univ. Press 1985.

[11] Goldstein, J. A. and J. Tervo: Existence results of solutions for a system of nonlinear partial differential equations related to bioreactors. Dyn. Syst. Appl. 5 (1996), $197-210$.

[12] Isidori, A.: Nonlinear Control Systems. Berlin: Springer 1989.

[13] Julien, S., Babary, J. P. and M. T. Nihtilä: On modelling of boundary conditions and estimation for fixed-bed bioreactors. Math. Modelling of Systems 1 (1995), $233-243$.

[14] Lions, J. L.: Optimal Control of Systems Governed by Partial Differential Equations. New York: Springer 1971 .

[15] Logemann, H. and H. Zwart: On robust PI-control of infinite-dimensional systems. SIAM J. Contr. Optim. 30 (1992), 573 - 593.

[16] Logemann, H. and S. Townley: Low-gain control of uncertain regular linear systems. SIAM J. Contr. Optim. 35 (1997), $78-116$.

[17] Nihtilä, M. T., Tervo, J. and J. P. Kaipio: Simulation of a nonlinear distributed parameter bioreactor by FEM approach. Simul. Practice and Theory 5 (1997), $199-216$.

[18] Pazy, A.: Semigroups of Linear Operators and Applications to Partial Differential Equations. Berlin: Springer 1983.

[19] Protter, M. H. and H. F. Weinberger: Maximum Principles in Differential Equations. Englewood Cliffs: Prentice-Hall 1967.

[20] Salmi, T. and J. J. Romanainen: A novel exit boundary condition for the axial dispersion model. Chem. Eng. and Proc. 34 (1995), 359 - 366.

[21] Tanabe, H.: Equations of Evolution. Pitman, 1979.

[22] 'Tervo, J. and M. T. Nihtilä: Stability for linearized control system related to a fixedbed bioreactor. Summary in paper form: J. Math. Systems, Estimation, and Control 8 (1998), 483 - 486; full paper (pp. 1 - 24) in electronic form: ftp://trick.ntp.springer.de/ pub/jmsec/49864.ps.gz.

[23] Tervo, J., Vauhkonen, M., Ronkanen, P. J. and J. Kaipio: A three-dimensional finite element model for the control of certain nonlinear bioreactors. Report. Kuopio: Dept. Appl. Phys. Univ., Report 4, 1996. 